\title{
A Review Study of Co-Extracting Opinion Targets and Opinion Words from Online Reviews
}

\author{
Saru \\ M.Tech Student \\ Dept. ofComputer \\ Science\&Engg, \\ Ajay Kumar Garg \\ Engineering College, \\ UPTU,Ghaziabad, India
}

\author{
Mamta Bhusry \\ Professor \\ Dept. ofComputer \\ Science\&Engg \\ Ajay Kumar Garg \\ Engineering College, \\ UPTU,Ghaziabad, India
}

\author{
Himani Singh \\ M.Tech Student \\ Dept. ofComputer \\ Science\&Engg, \\ Ajay Kumar Garg \\ Engineering College, \\ UPTU,Ghaziabad, India
}

\begin{abstract}
With the rapid development of e-trade, more items are sold on the Web, thus numerous individuals are additionally acquiring items on the web. With a specific end goal to upgrade consumer loyalty and shopping background, it has turned into a typical training for online shippers to empower their clients to survey or to express reviews on the items that they have bought. With considerable number of normal clients receiving to be good with the Web furthermore an expanding number of clients are composing reviews. In this research work we exhibit survey investigation of the existing co-separating algorithms is utilized to concentrate opinion targets and sentiment words. This paper also displays an investigation of existing co-extracting algorithm and models are utilized to concentrate opinion targets and opinion words
\end{abstract}

\section{Keywords}

Co-extracting algorithm, opinion targets, opinion words, ecommerce and co-extracting model

\section{INTRODUCTION}

just In recent years, mining opinions and analyzing sentiments viewpoints in online reviews gets to be helpful and has pulled in a considerable measure of considerations from numerous scientists. In opinion mining, one major issue is to concentrate opinion targets, which are characterized as the items on which clients have communicated their opinions, regularly as things or thing phrases. This task is critical in light of the fact that clients are normally not fulfilled by simply the general estimation extremity of an item; however hope to locate the fine-grained assumptions around a viewpoint or an item feature specified in surveys [1] To satisfy this assignment, existing concentrates as a rule viewed opinion words as concrete pointers. This technique depends on the perception that opinion words are typically used to change opinion targets, and there are opinion relations and relationship between them. For instance, "magnificent" and "incredible" are typically used to change "plan" about wireless in reviews, so there are compact relationship between them. If "superb"" and "phenomenal" had been known not opinion words, "configuration" is liable to be an opinion target in this area. In the intervening the extracted opinion targets can be utilized to extend more o.pinion words [2]

With the rapid extension of e-trade, more items are sold on the Web, thus numerous individuals are likewise buying items on the web. Considering the end goal to upgrade consumer loyalty and shopping knowledge, it has turned into a typical practice for online shippers to empower their clients to survey or to express reviews on the items that they have acquired. With huge number of basic clients receiving to be satisfactory with the Web additionally an expanding number of clients are composing reviews. Subsequently, item gets the more prominent number of reviews and due to this some wellknown items can get many reviews everywhere business locales [3], [4]. Besides, numerous surveys are extensive and have just a couple sentences containing reviews related item. This makes it hard to settle on a learned choice on whether to purchase the item or not for a potential client in the wake of understanding them.

Removing opinion targets and sentiment words from online reviews are two key errands in conclusion mining. Numerous research works proposes way to deal with by and large concentrate them with chart co-ranking.

- In past strategies, mining the opinion relations between sentiment targets and conclusion words was the way to collective extraction. To this end, the most embraced strategies have been closest neighbor rules and syntactic examples.

- Nearest neighbor rules respect the closest descriptive word/verb to a thing/thing phrase in a constrained frame as its modifier.

- Syntactic data, in which the opinion relations among words are, chose by reliance relations in the parsing tree.

\section{BASIC STRUCTURE}

Concentrated on opinion target extraction, for example, [1]; [2]; [3]; [4]; [5], can be partitioned into two primary classes: directed and unsupervised strategies. In administered approaches, the opinion target extraction task was generally viewed as a succession marking task [5]; [6]; [7]; [8]. The principle restriction of these strategies is that marking preparing information for every space is monotonous and impracticable. In unsupervised strategies, like our own, most methodologies viewed opinion words as the vital markers for opinion targets [1] [3]. It composed some syntactic examples to concentrate opinion targets [9]. A Double Propagation technique is proposed to extend notion words and opinion targets iteratively, where they likewise misused syntactic relations between words. The fundamental constraint of Qiu's technique is that the examples taking into account reliance parsing tree might present numerous commotions for the substantial quantities.

In [10] expanded Qiu's strategy. Other than the examples utilized as a part of Qiu's strategy, they contained some other exceptional outlined examples to expand review. Moreover they utilized the HITS algorithm to process conclusion target confidences to enhance the exactness. In [11] is like above technique; they utilize a totally unsupervised WTM to catch 
opinion relations in sentences. At that point the opinion targets were separated in a standard arbitrary walk system where two components were considered: opinion significance and target significance

\section{CO-EXTRACTING APPROACHES}

There are numerous studies done on the extracting of opinion words and opinion targets. These are as follows:

Minqing $\mathrm{Hu}$ and Bing Liu (2004) studied the basic sorts of review configurations viz. (1) Pros and Cons (2) Upsides and downsides with free arrangement; they utilized the affiliation principle mining to remove the item include if the review fits in with either arrange 2 or organization (3) They utilized a regulated digging strategy for removing the item feature if the review is in Pros and Cons design. The extremity of the distinguished sentiment words was resolved utilizing the Word-Net. The sentence level sentiment introduction was chosen in view of the vast introduction. This work just concern with positive and negative introductions that uses set of seed descriptive words. Fundamental target of this framework is to give a component based rundown of countless reviews of items that are sold on the web. [2]

Robert C. Moore (2005), this research work depicts discriminative methodology. For the preparation of basic word arrangement models as it having exactness contrasted with more confusing generative models. If the words have a solid positive affiliation then the LLR score for a couple of words is high. Unlinked word feature checks the aggregate number of unlinked words [15].

Ana-Maria Popescu and Oren Etzioni (2005) added to an unsupervised data extraction framework named "OPINE". They decided the semantic introduction of words in light of another marking system called "unwinding naming". The likelihood of an item name is evaluated utilizing a redesign mathematical statement as a part of an iterative way. The unwinding naming algorithm stops when the worldwide name task stays consistent. The semantic introduction (SO) of words was figured taking into account the area features. They recognized the extremity of the conclusion phrases in view of the SO name doled out to the head words in them. "Know It All" framework is utilized to assemble the conclusion [4].

Xiaowen Ding et al (2008) proposed a strategy for "substance release" (recognizing the element if the name is accessible in the review) utilizing consecutive example mining and "element task" utilizing relative sentences mining. This technique is entirely helpful when a commentator is commenting on various items in the same review. In this technique express and verifiable opinions are additionally considered. This framework primarily centered on item included for synopsis of review. Object feature, opinion extraction and conclusion extremity identification are the errands figured to propose another machine learning with Conditional Random Fields (CRFs). Impact of elements for survey mining is broke down. CRF can without much of section incorporate different elements than the Lexicalized HMM model [3].

G. Qiu, et. al. (2009) proposed a framework for distinguishing the favored elements in relative sentences (PCS). Their goal was to distinguish the favored substance of the creator in a near sentence such as "Camera $\mathrm{X}$ is better than Camera Y". Their research depended on the perception of near and superlative words in the English dialect. They dealt with the blends of near words $(\mathrm{C})$ and components $(\mathrm{F})$ in the accompanying cases and comparing principles were created.
$\mathrm{C}$ is determined - In a relative sentence, if $\mathrm{C}$ has a positive introduction then the substance that precedes $\mathrm{C}$ is the preferred or generally the element that comes after $\mathrm{C}$.

$\mathrm{C}$ is not determined but rather $\mathrm{F}$ is inflexible

\section{$\mathrm{C}$ and $\mathrm{F}$ both are not determined}

$\mathrm{C}$ is an element marker

A sum of 837 near sentences were tried and with $94.4 \%$ of exactness. They proposed another measure, the one-side affiliation (OSA) measure for deciding the level of relationship between the similar word and the element feature. In this research work, double producing that is statement language and separated item components are additionally proposed. In this research work, Surface dependence analyzing is utilized to extricate the opinion words. In this one expression is avoided as head and others are needy. Name is utilized to declare to the connection [10]. Yuanbin et. al. (2009), In this research work creators decided conclusion mining from unstructured archives. To concentrate relations between item elements and opinion expressions phrase reliance tree is built. It utilizes bit capacity to assemble phrase reliance trees. In this research work, opinion unit contains an item feature, a statement of opinion, and an enthusiastic mentality. These are valuable in opinion mining task. Components are separated utilizing phrase reliance tree. Tree parts (SVM-W Tree and SVM-P Tree) are additionally deduced in this research work [5]. Qi Zhang, Yuanbin Wu, Tao Li (2009), authors were contrasting the exhibitions of various connection extraction strategies on the corpus. Treeportion based methodology is proposed. The aftereffect of this framework depends on SVM. It assesses two sorts of piece capacities: 1) straight and 2) outspread premise capacity. This framework contrasts diverse extraction relations and database which having a few surveys of cellular telephones and camera. Diverse techniques are utilized to perform for various database or quantity [16]. Tengfei Ma Xiaojun Wan (2010), research work presents Centering Theory. In this research work, creators used relevant data for extricating the objective framework. This research work extricates an express and understood sentiment focuses from news remarks with the assistance of Centering Theory. "Focuses" is the elements serving to interface. Forward-looking focus, Backwardlooking focus, Rank and so on is characterized in this research work [8].

Zhu Jian et al (2010) proposed another technique in view of artificial neural networks (ANN) named as "i-model". In this research work, w machine learning structure is proposed. It depends on Conditional Random Fields (CRFs) is utilized utilize rich components for eliminating object features, positive opinions and negative opinions. The notable opinion components are organized considering a score and feature weight is computed [13].

Zhang Lei and Bing Liu (2010) proposed a technique to recognize the things item includes that is valuable for identifying the conclusions. They recommended that, the review measure of the order assignment can be enhanced by examining the thing features. The proposed algorithm manages the issues of the best in class double propagation technique which is utilized for feature extraction. In this research work feature extraction and feature ranking methodologies are proposed to manage the issue of coextracting the conclusion. In this element candidates are located with its significance. A HIT algorithm is utilized to 
rates Web pages furthermore to acquire the element significance ranking [11]

Qiu et al (2010) underlined the significance of opinion dictionary development and opinion target extraction for the reason successful estimation research. The algorithm initiates with a seed word and distinguishes the related target and maintains recognizing the other available opinion words and motivations until not any more new opinion words or targets can be included. With the end goal of syntactic feature POS labeling, they utilized the Stanford POS labeling device and for parsing the sentences, they utilized the "Minibar" sentence parser. They considered opinion words to be descriptors and focuses to be things or thing phrases:

Qiu et al (2010) underlined the significance of opinion dictionary development and opinion target extraction for the reason successful estimation research. The algorithm initiates with a seed word and distinguishes the related target and maintains recognizing the other available opinion words and motivations until not any more new opinion words or targets can be included. With the end goal of syntactic feature POS labeling, they utilized the Stanford POS labeling device and for parsing the sentences, they utilized the "Minibar" sentence parser. They considered opinion words to be descriptors and focuses to be things or thing phrases:

1. Extricating targets utilizing the opinion words

2. Extricating targets utilizing the separated targets

3. Extricating opinion words utilizing the separated targets

4. Extricating opinion words utilizing both the given and separated opinion words.

This methodology brought about an impressive change in accuracy, review and F-score over the beforehand accessible methodologies for sentiment words and conclusion target ID. They likewise beat other cutting edge techniques. This framework does not required extra assets. A beginning seed conclusion dictionary is utilized to remove the opinion. This framework removes opinion words from past emphases opinion words and after that objectives it through the ID of syntactic relations. Connection Identification is utilized to recognize the connection between opinion words and targets words. [6]

B. Wang and H. Wang (2011), this research work utilized the recipe of shared data to device the relationship since common data of a low reappearance word pair has a tendency to be high. In this framework shared data is utilized to quantify the affiliation. Components are altered with the assistance of encompassing sentiment words. Components are only the thing, expressions and sentences in the record. In this framework item feature and opinion words are joined together. It depends on connection reliance property. [14]

Zhao et al (2012) proposed a technique conquer the constraints of the dual production strategy for feature unique proof in substantial and little corpora. The double propagation strategy results in low exactness and low review for extensive and little quantities. They have handled the low review issue by presenting "part-entire example" and "no example". The low exactness issue is overcome by utilizing feature importance and feature ranking [12].

Fangtao Li et. al (2012), this research work proposes space adjustment system. It is utilized for estimation and subject language co-extraction in a space of hobby. They utilized
Relational Adaptive Process (RAP) algorithm to extend the seeds in the objective area. In the objective space highcertainty assumption and point seeds are produced. Processing (RAP) extend the seeds in the objective area. Assumption and theme dictionary co-extraction is twofold structure. [7]

\section{COMPARATIVE ANALYSIS OF PREVIOUS APPROACHES}

Below table represents the comparative analysis of approaches described in review part of this paper:

Table I: Comparative analysis of existing approaches

\begin{tabular}{|c|c|c|}
\hline $\begin{array}{l}\text { Author/Ye } \\
\text { ar }\end{array}$ & $\begin{array}{l}\text { Name of } \\
\text { Research }\end{array}$ & Research approach \\
\hline $\begin{array}{l}\text { R. C. } \\
\text { Moore } \\
(2005)\end{array}$ & $\begin{array}{l}\text { A } \\
\text { discriminati } \\
\text { ve } \\
\text { framework } \\
\text { for bilingual } \\
\text { word } \\
\text { alignment }\end{array}$ & $\begin{array}{l}\text { In this research work the Log- } \\
\text { Likelihood-Based Model is } \\
\text { utilized to gauge word } \\
\text { affiliation. If the words have a } \\
\text { solid positive affiliation then } \\
\text { the LLR score for a couple of } \\
\text { words is high. }\end{array}$ \\
\hline $\begin{array}{l}\text { X. Ding, } \\
\text { B. Liu, } \\
\text { and P. S. } \\
\text { Yu, } \\
(2008)\end{array}$ & $\begin{array}{l}\text { A holistic } \\
\text { lexicon- } \\
\text { based } \\
\text { approach to } \\
\text { opinion } \\
\text { mining }\end{array}$ & $\begin{array}{l}\text { Proposed a strategy for } \\
\text { disclosure" } \\
\text { "substance } \\
\text { (recognizing the element if the } \\
\text { name is accessible in the } \\
\text { review) utilizing consecutive } \\
\text { example mining and "element } \\
\text { task" (when pronouns are } \\
\text { utilized to speak to the element) } \\
\text { utilizing relative sentences } \\
\text { mining. }\end{array}$ \\
\hline $\begin{array}{l}\text { Q. Zhang, } \\
\text { Y. Wu, T. } \\
\text { Li, M. } \\
\text { Ogihara, } \\
\text { J. } \\
\text { Johnson, } \\
\text { and X. } \\
\text { Huang, } \\
(2009)\end{array}$ & $\begin{array}{l}\text { Mining } \\
\text { product } \\
\text { reviews } \\
\text { based on } \\
\text { shallow } \\
\text { dependency } \\
\text { parsing }\end{array}$ & $\begin{array}{l}\text { In this research work, creators } \\
\text { were contrasting the exhibitions } \\
\text { of various connection } \\
\text { extraction strategies on the } \\
\text { corpus. Tree-portion based } \\
\text { methodology is proposed. }\end{array}$ \\
\hline $\begin{array}{l}\text { T. Ma and } \\
\text { X. Wan, } \\
(2010)\end{array}$ & $\begin{array}{l}\text { Opinion } \\
\text { target } \\
\text { extraction in } \\
\text { Chinese } \\
\text { news } \\
\text { comments }\end{array}$ & $\begin{array}{l}\text { This research work extricates } \\
\text { an express and understood } \\
\text { sentiment focuses from news } \\
\text { remarks with the assistance of } \\
\text { Centering Theory. }\end{array}$ \\
\hline $\begin{array}{ll}\text { F. Li, } & \text {. } \\
\text { Han, } & \text { M. } \\
\text { Huang, } & \text { X. } \\
\text { Zhu, } & \text { Y. } \\
\text { Xia, } & \text { S. } \\
\text { Zhang, } & \\
\text { and } & \text { H. } \\
\text { Yu, } & \\
(2010) & \\
\end{array}$ & $\begin{array}{l}\text { Structure- } \\
\text { aware } \\
\text { review } \\
\text { mining and } \\
\text { summarizati } \\
\text { on }\end{array}$ & $\begin{array}{l}\text { Proposed another technique in } \\
\text { iew of counterfeit neural } \\
\text { pystems (ANN) named as "i- } \\
\text { nodel". In this research work, w } \\
\text { nachine learning structure is } \\
\text { roposed. }\end{array}$ \\
\hline
\end{tabular}




\begin{tabular}{|c|c|c|}
\hline $\begin{array}{l}\text { Zhang Lei } \\
\text { and Bing } \\
\text { Liu } \\
(2010)\end{array}$ & $\begin{array}{l}\text { Extracting } \\
\text { and ranking } \\
\text { product } \\
\text { features in } \\
\text { opinion } \\
\text { documents } \\
\end{array}$ & $\begin{array}{l}\text { In this element hopefuls are } \\
\text { positioned with its significance. } \\
\text { A HIT algorithm is utilized to } \\
\text { rates Web pages furthermore to } \\
\text { acquire the element } \\
\text { significance ranking. }\end{array}$ \\
\hline $\begin{array}{l}\text { GuangQiu } \\
\text { et al } \\
(2010)\end{array}$ & $\begin{array}{l}\text { Opinion } \\
\text { word } \\
\text { expansion } \\
\text { and target } \\
\text { extraction } \\
\text { through } \\
\text { double } \\
\text { propagation }\end{array}$ & $\begin{array}{l}\text { They proposed a spread } \\
\text { algorithm for distinguishing } \\
\text { sentiment words and the related } \\
\text { targets. }\end{array}$ \\
\hline $\begin{array}{l}\text { B. Wang } \\
\text { and H. } \\
\text { Wang, } \\
(2011)\end{array}$ & $\begin{array}{l}\text { Bootstrappin } \\
\mathrm{g} \text { both } \\
\text { product } \\
\text { features and } \\
\text { opinion } \\
\text { words from } \\
\text { Chinese } \\
\text { customer } \\
\text { reviews with } \\
\text { cross } \\
\text { inducing }\end{array}$ & $\begin{array}{l}\text { This research work utilized the } \\
\text { recipe of shared data to gauge } \\
\text { the relationship since common } \\
\text { data of a low recurrence word } \\
\text { pair has a tendency to be high. }\end{array}$ \\
\hline $\begin{array}{l}\text { Zhao et al } \\
(2012)\end{array}$ & $\begin{array}{l}\text { Opinion } \\
\text { target } \\
\text { extraction } \\
\text { using word } \\
\text { based } \\
\text { translation } \\
\text { model }\end{array}$ & $\begin{array}{l}\text { Proposed a technique conquer } \\
\text { the constraints of the twofold } \\
\text { proliferation strategy for } \\
\text { feature distinguishing proof in } \\
\text { substantial and little corpora }\end{array}$ \\
\hline $\begin{array}{l}\text { Fangtao } \\
\text { Li, } \\
\text { SinnoJiali } \\
\text { n Pan, Ou } \\
\text { Jin, Qiang } \\
\text { Yang and } \\
\text { XiaoyanZ } \\
\text { hu (2012) }\end{array}$ & $\begin{array}{l}\text { Cross- } \\
\text { domain co } \\
\text { extraction of } \\
\text { sentiment } \\
\text { and topic } \\
\text { lexicons }\end{array}$ & $\begin{array}{l}\text { This research work proposes } \\
\text { space adjustment system. It is } \\
\text { utilized for estimation and } \\
\text { subject vocabulary co- } \\
\text { extraction in main of interest. }\end{array}$ \\
\hline
\end{tabular}

\section{CONCLUSIONS}

In this research work, we have reviewed a number of methodologies utilized as a part of co-extricating of opinion words and conclusion focuses from surveys. From the survey review, it is obvious that the field of viewpoint research is developing consistently as it has huge potential for business insight related applications. Despite the fact that a ton of opinion investigation techniques have been proposed and worked on utilizing the directed, unsupervised and semiadministered learning and grouping algorithms, still there is a tremendous potential for growing new strategies for estimation research at the archive level.

\section{REFERENCES}

[1] A. Mukherjee and B. Liu, (Jul. 2012). "Modeling review comments," in Proc. 50th Annu. Meeting Assoc. Computer. Linguistics, Jeju, Korea, pp. 320-329.

[2] Xiaowen Ding, Bing Liu, and Philip S. Yu, (2008). "A holistic lexicon-based approach to opinion mining". In Proceedings of the Conference on Web Search and Web Data Mining (WSDM).

[3] Ana-Maria Popescu and Oren Etzioni, (2005). "Extracting product features and opinions from reviews". In Proceedings of the conference on Human Language
Technology and Empirical Methods in Natural Language Processing, HLT '05, pages 339-346, Stroudsburg, PA, USA, Association for Computational Linguistics.

[4] Yuanbin Wu, Qi Zhang, Xuanjing Huang, and LideWu, (2009). "Phrase dependency parsing for opinion mining". In EMNLP, pages 1533-1541. ACL.

[5] Wei Jin and Hay Ho Huang, (2009).“A novel lexicalized hmm-based learning framework for web opinion mining". In Proceedings of International Conference on Machine Learning (ICML), 2009.

[6] Fangtao Li, Chao Han, Minlie Huang, Xiaoyan Zhu, Yingju Xia, Shu Zhang, and Hao Yu, (2010). "Structureaware review mining and summarization". In Chu-Ren Huang and Dan Jurafsky, editors, COLING, pages 653661. Tsinghua University Press, 2010.

[7] Tengfei Ma and Xiaojun Wan,(2010)." Opinion target extraction in Chinese news comments". In Chu- Ren Huang and Dan Jurafsky, editors, COLING (Posters), pages782-790. Chinese Information Processing Society of China, 2010

[8] Mingqin $\mathrm{Hu}$ and Bing Liu, (2004a). "Mining opinion features in customer reviews". In Proceedings of Conference on Artificial Intelligence (AAAI), 2004.

[9] GuangQiu, Bing Liu, Jiajun Bu, and Chun Che, (2009). "Expanding domain sentiment lexicon through double propagation". 2009.

[10] Lei Zhang, Bing Liu, Suk Hwan Lim, and Eamonn O'Brien-Strain, (2010). "Extracting and ranking product features in opinion documents". In Chu-Ren Huang and Dan Jurafsky, editors, COLING (Posters), pages 1462 1470. Chinese Information Processing Society of China, 2010.

[11] Kang Liu, Liheng Xu, and Jun Zhao, (2012). "Opinion target extraction using word-based translation model". In Proceedings of the 2012 Joint Conference on Empirical Methods in Natural Language Processing and Computational Natural Language Learning, pages 13461356, Jeju Island, Korea, July 2012. Association for Computational Linguistics.

[12] Li, S. J. Pan, O. Jin, Q. Yang, and X. Zhu, (2012) "Cross-domain co extraction of sentiment and topic lexicons," in Proc. 50th Annu. Meeting Assoc. Comput. Linguistics, Jeju, Korea, pp. 410-419.

[13] B. Wang and H. Wang, (2011) "Bootstrapping both product features and opinion words from Chinese customer reviews with crossinducing," in Proc. 3rd Int. Joint Conf. Natural Lang. Process.,

[14] R. C. Moore, (2005) "A discriminative framework for bilingual word alignment," in Proc. Conf. Human Lang. Technol. Empirical Methods Natural Lang. Process., Vancouver, BC, Canada, 2005, pp. 81-88.

[15] Q. Zhang, Y. Wu, T. Li, M. Ogihara, J. Johnson, and X. Huang, (2009) "Mining product reviews based on shallow dependency parsing," in Proc. 32nd Int. ACM SIGIR Conf. Res. Develop. Inf. Retrieval, Boston, MA, USA, 2009, pp. 726-727. 